\title{
Editorial
}

\section{Sensors for Coastal Monitoring}

\section{Francesco Serafino, ${ }^{1}$ Jochen Horstmann, ${ }^{2}$ José Carlos Nieto Borge, ${ }^{3}$ Claudio Lugni, ${ }^{4}$ and Maurizio Brocchini ${ }^{5}$}

\author{
${ }^{1}$ Institute for Electromagnetic Sensing of the Environment, CNR, Via Diocleziano 328, 80124 Naples, Italy \\ ${ }^{2}$ Institute of Coastal Research, Helmholtz-Zentrum Geesthacht, Max-Planck-Straße, 121502 Geesthacht, Germany \\ ${ }^{3}$ Universidad de Alcalá, Plaza de San Diego, s/n, 28801 Alcalá de Henares, Spain \\ ${ }^{4}$ Marine Technology Research Institute, CNR, Via di Vallerano 139, 00128 Rome, Italy \\ ${ }^{5}$ DICEA, Università Politecnica delle Marche, Via Brecce Bianche 12, 60131 Ancona, Italy
}

Correspondence should be addressed to Francesco Serafino; serafino.f@irea.cnr.it

Received 3 March 2016; Accepted 3 March 2016

Copyright (c) 2016 Francesco Serafino et al. This is an open access article distributed under the Creative Commons Attribution License, which permits unrestricted use, distribution, and reproduction in any medium, provided the original work is properly cited.

The use of sensors for coastal monitoring is, nowadays, a very common and widespread practice due to their flexibility and capability of providing accurate and heterogeneous measurements of parameters that characterize the marine environment, even in the coastal area. Indeed, the capability of measuring coastal phenomena (waves, currents, plumes, morphology, etc.) in real time and with high accuracy makes these sensors extremely useful in improving the comprehension of the dynamic evolution of coastal phenomena. Examples are the proper understanding of the effects of severe weather conditions on the coastal morphodynamics and the real-time monitoring of the spill of pollutants.

In this theme issue, we have collected a series of works from international experts in their field, which describe advanced and innovative systems for coastal and riverine monitoring.

In the past, some significant works have summarized the state of the art in this field $[1,2]$. However, the exponential growth of technology continuously provides new inputs and challenges towards the use of innovative and more accurate sensors, motivating the present issue. For the same reason, we have given main emphasis to the capability of the sensor technology rather than to the originality of the scientific contribution.

The paper entitled "Optimal Fair Scheduling in S-TDMA Sensor Networks for Monitoring River Plumes" proposes a scheduling procedure to obtain the optimal fair frame at the gateway of an underwater wireless sensor network to be used in the monitoring of river plumes. Focus is on finding the theoretical maximum throughput by overlapping the transmissions of the nodes while keeping a balanced received data rate from each sensor of the network. Optimal performances are achieved if the gateway is at the center of the network; these decrease to $70 \%$ of the maximum normalized throughput if the gateway is at the edge of the network.

An investigation about a multifocus color image fusion algorithm based on nonsubsampled shearlet transform (NSST) and pulsed coupled neural networks (PCNN) is carried out in the contribution titled "Multifocus Color Image Fusion Based on NSST and PCNN." The proposed algorithm is tested over a set of image couples and other, well assessed, algorithms, known in literature, are employed as benchmark; the proposed algorithm outperforms the traditional ones in terms of fusion performance.

The X-band radar technology has significantly contributed to providing a new insight in the real-time monitoring of sea waves, improving the ship navigation safety and the comprehension of medium-scale hydrodynamic phenomena.

The paper entitled "Visibility of River Plume Fronts with an X-Band Radar" investigates the opportunity to detect river plume fronts from marine X-band radar images. To this aim, a huge radar dataset acquired close to the mouth of the Tenryu river, in Japan, has been compared with a number of satellite and in situ datasets to prove the visibility of plume fronts in radar acquisitions. In addition, the author provides the results of a statistical analysis, which involves the "intensity" of river 
plume fronts in radar data, the local wind vector, and the local water level, to assess the "detectability" of the plume fronts as function of the environmental conditions.

The published paper "Application of X-Band Wave Radar for Coastal Dynamic Analysis: Case Test of Bagnara Calabra (South Tyrrhenian Sea, Italy)" illustrates the applicability of conventional X-band marine radars to analyse the behaviour of waves in coastal waters. The analysis carried out in this work permits identifying the spatiotemporal evolution of incoming waves in coastal waters, including the local wave height, as well as identifying the rip currents as a result of the analysis. Using a different remote sensing sensor, the paper "Monitoring Individual Wave Characteristics in the Inner Surf with a 2-Dimensional Laser Scanner (LiDAR)" shows the use of a two-dimensional laser scanner (LiDAR), which operates in the optical domain of the electromagnetic spectrum, rather than the X-band, which works in the microwave range, to derive individual wave features and their temporal evolution. The work has been applied in the inner surf and swash zones of a microtidal beach. Parameters, such as wave height, wave periods, and wave celerity, are obtained with this technique. The paper "LG-Mod: A Modified Local Gradient (LG) Method to Retrieve SAR Sea Surface Wind Directions in Marine Coastal Areas" applies the Synthetic Aperture Radar system on board the ESA-Envisat satellite (ASAR) to derive wind directions in coastal areas. The proposed method to retrieve winds is based on the computation of local gradients over quasilinear and quasiperiodic structures detected by SAR imagery. The proposed wind estimation model is validated against in situ coastal wind measurements provided by anchored buoys with 63 Envisat ASAR images.

In the paper "GNSS-R Delay-Doppler Map Simulation Based on the 2004 Sumatra-Andaman Tsunami Event," a new method for simulating Global Navigation Satellite SystemReflectometry Doppler maps is presented to analyse tsunamidominant sea surfaces. The technique uses a Cox and Munk based model. The feasibility of Cox and Munk model under the tsunami scenario is compared with measurements having a correlation coefficient of 0.93 . The analysis carried out in the work may permit retrieving in the future tsunami parameters using the techniques explained in the paper. Using different techniques, the paper "A New Small Drifter for Shallow Water Basins: Application to the Study of Surface Currents in the Muggia Bay (Italy)" uses Lagrangian drifters to analyse surface currents for coastal areas under different meteorological conditions. This paper describes a small drifter prototype that transmits its GPS location via GSM phone network. From the trajectories of the drifters, the local currents are derived; that analysis includes additional information, such as the surface circulation.

The manuscript "Wave Height Estimation from Shipborne X-Band Nautical Radar Images" describes some improvements of a shadowing-analysis-based algorithm for the wave height estimation from X-band radar images acquired from a radar on a moving ship. The algorithm involves first the extraction of the shadowed areas from radar images through an edge detection technique. Then, using the calculated illumination ratios in local areas, the root mean square (RMS) surface slope is derived by a curve fitting Smith's function. Finally, the significant wave height is estimated from the RMS surface slope and the average zero-crossing wave period.

This theme issue is a good opportunity for the interested readers to be updated on the most recent results of the research in the field of coastal monitoring achieved and described by the researchers who contributed to such research.

\section{Francesco Serafino Jochen Horstmann José Carlos Nieto Borge Claudio Lugni Maurizio Brocchini}

\section{References}

[1] T. J. Malthus and P. J. Mumby, "Remote sensing of the coastal zone: an overview and priorities for future research," International Journal of Remote Sensing, vol. 24, no. 13, pp. 2805-2815, 2003.

[2] V. Klemas, "Remote sensing of coastal and ocean currents: an overview," Journal of Coastal Research, vol. 28, no. 3, pp. 576586, 2012. 


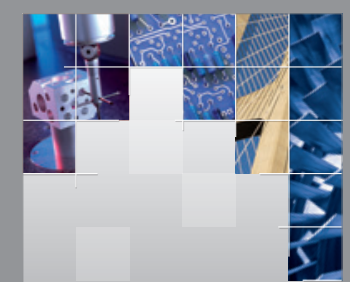

\section{Enfincering}
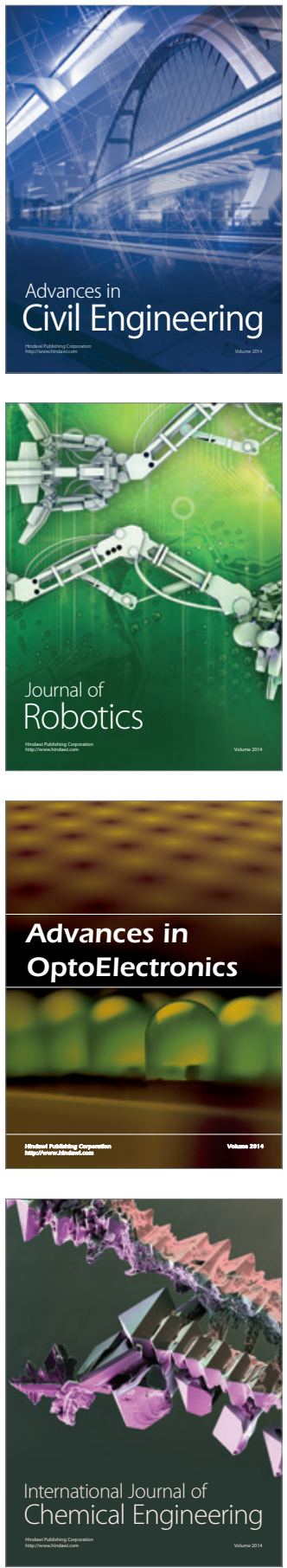

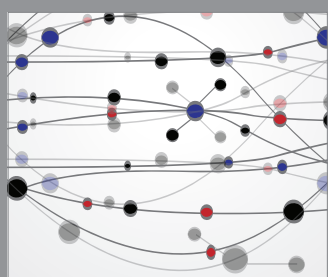

The Scientific World Journal

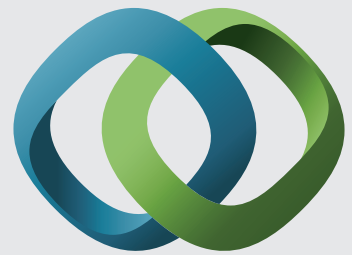

\section{Hindawi}

Submit your manuscripts at

http://www.hindawi.com
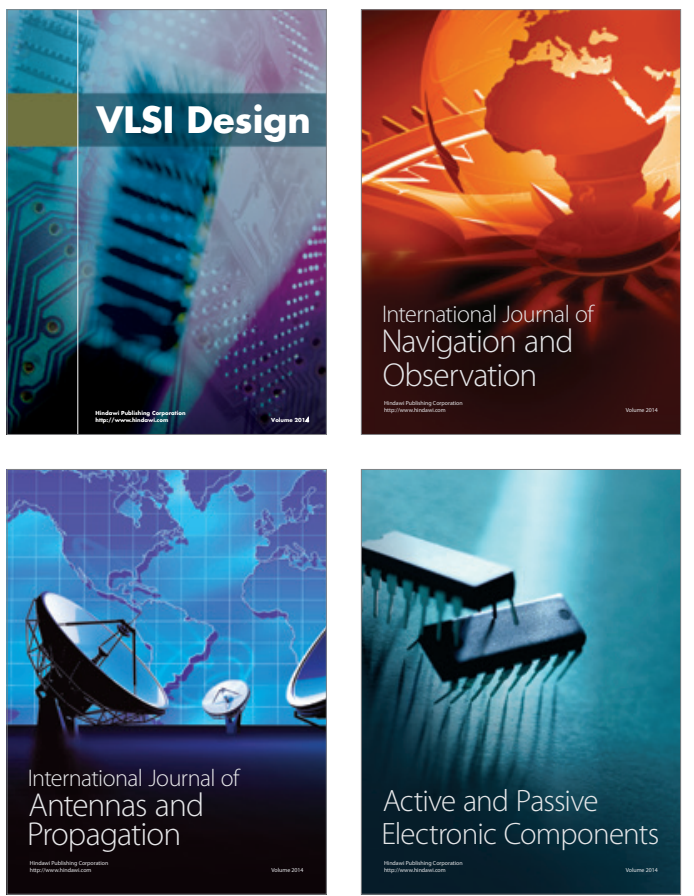
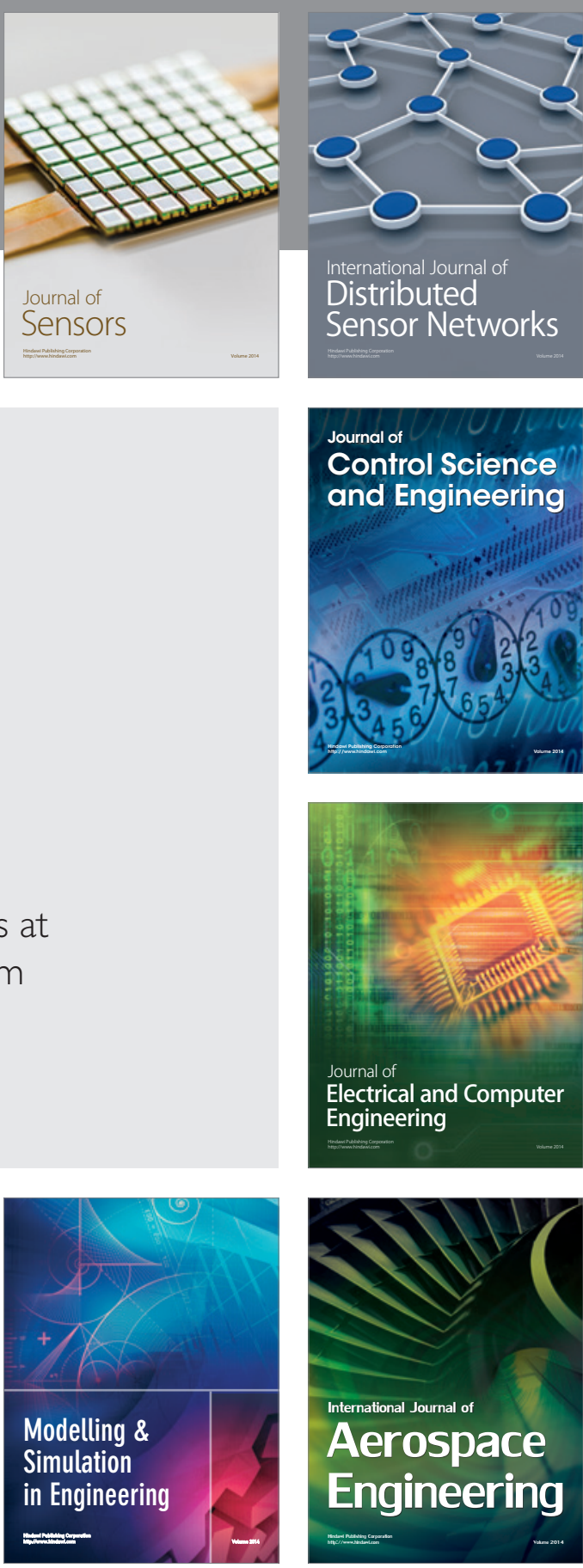

International Journal of

Distributed

Sensor Networks

Journal of

Control Science

and Engineering
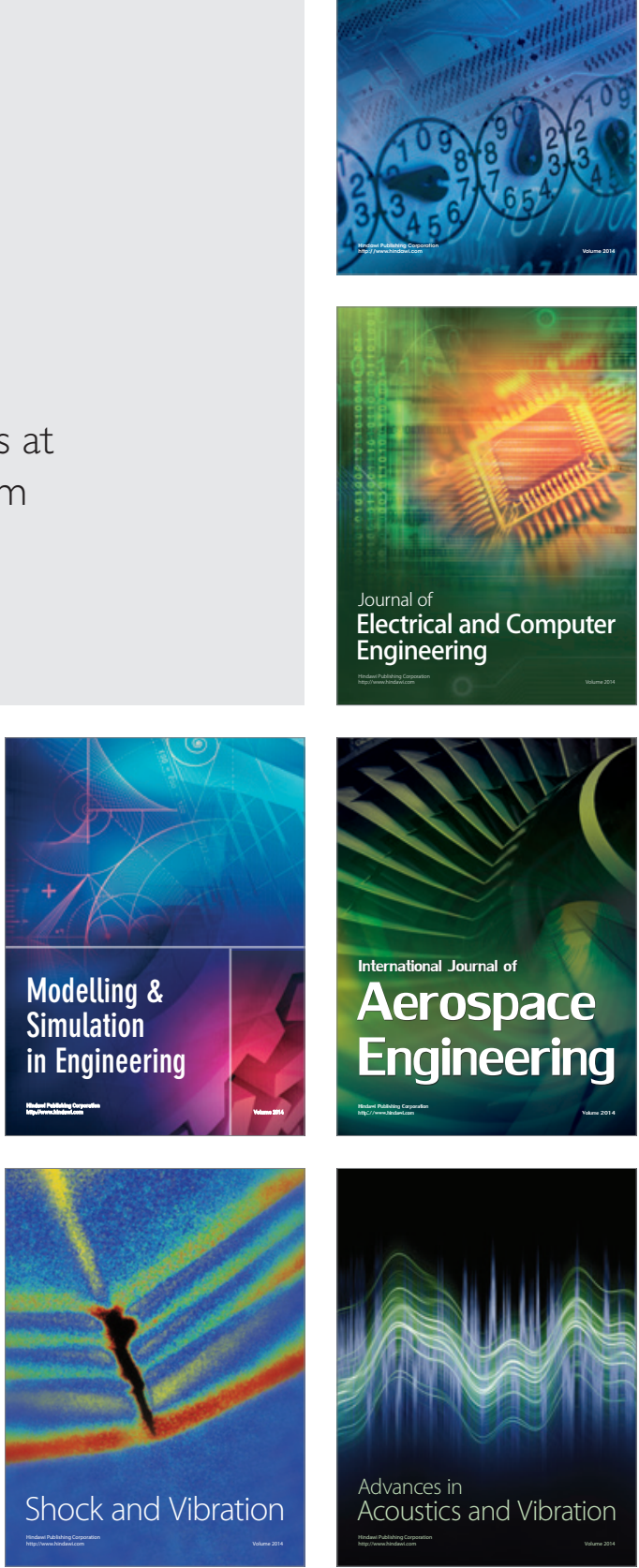